\title{
Deep Learning in Multimedia Forensics
}

\author{
Luisa Verdoliva \\ University Federico Ii of Naples \\ Naples, Italy \\ verdoliv@unina.it
}

\begin{abstract}
With the widespread diffusion of powerful media editing tools, falsifying images and videos has become easier and easier in the last few years. Fake multimedia, often used to support fake news, represents a growing menace in many fields of life, notably in politics, journalism, and the judiciary. In response to this threat, the signal processing community has produced a major research effort. A large number of methods have been proposed for source identification, forgery detection and localization, relying on the typical signal processing tools.
\end{abstract}

The advent of deep learning, however, is changing the rules of the game. On one hand, new sophisticated methods based on deep learning have been proposed to accomplish manipulations that were previously unthinkable. On the other hand, deep learning provides also the analyst with new powerful forensic tools. Given a suitably large training set, deep learning architectures ensure usually a significant performance gain with respect to conventional methods, and a much higher robustness to post-processing and evasions.

In this talk after reviewing the main approaches proposed in the literature to ensure media authenticity, the most promising solutions relying on Convolutional Neural Networks will be explored with special attention to realistic scenarios, such as when manipulated images and videos are spread out over social networks. In addition, an analysis of the efficacy of adversarial attacks on such methods will be presented.

\section{CCS Concepts/ACM Classifiers}

- Surveys and overviews Security and Privacy

\section{Author Keywords}

Multimedia forensics; forgery detection and localization; deep learning; convolutional neural networks.

\section{BIOGRAPHY}

Dr. Luisa Verdoliva is Assistant Professor in the Department of Electrical Engineering and Information Technology at University Federico II of Naples and holder of the National Habilitation for Associate Professor in Telecommunications. Her research activity focuses on deep learning for multimedia forensics, in particular on source identification, image and video forgery detection and localization. She is member of the IEEE Information Forensics and Security Technical Committee and Associate Editor for IEEE Transactions on Information Forensics and Security. She has been serving on the technical program committees of numerous image processing and digital forensics conferences. She is the Principal Investigator for the Research Unit of University Federico II of Naples in the DISPARITY (Digital, Semantic and Physical Analysis of Media Integrity) project funded by DARPA (Defense Advanced Research Projects Agency) under the MEDIFOR program. She led her research group in several international contests, including the recent 2018 IEEE Signal Processing Cup on camera model identification (first prize) and the 2013 IEEE Image Forensics Challenge (first prize both in the detection and localization task).

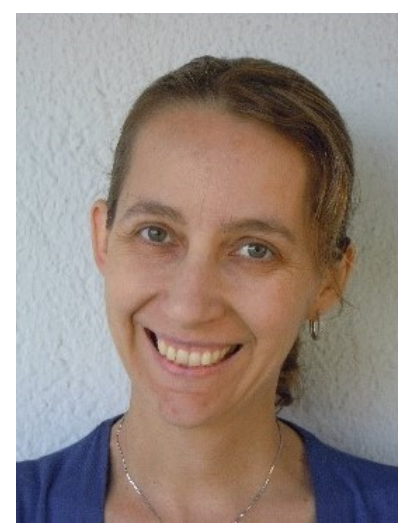

Permission to make digital or hard copies of part or all of this work for personal or classroom use is granted without fee provided that copies are not made or distributed for profit or commercial advantage and that copies bear this notice and the full citation on the first page. Copyrights for third-party components of this work must be honored. For all other uses, contact the Owner/Author. IH\&MMSec '18, June 20-22, 2018, Innsbruck, Austria.

(C) 2018 Copyright is held by the owner/author(s).

ACM ISBN 978-1-4503-5625-1/18/06.

DOI: https://doi.org/10.1145/3206004.3206024 